\title{
Recognizing TORCH Group of Infections on Fetal Sonography
}

\author{
${ }^{1}$ Sandesh Subramanya, ${ }^{2}$ Bhargavi Patham, ${ }^{3}$ Sanja Plavsic Kupesic \\ ${ }^{1}$ Instructor, Department of Biomedical Sciences, Center of Excellence for Infectious Diseases, Paul L Foster School of Medicine \\ Texas Tech University Health Sciences Center, El Paso, Texas 79905, USA \\ ${ }^{2}$ Assistant Professor of Pathophysiology and Cell Biology, Department of Medical Education, Paul L Foster School of Medicine \\ Texas Tech University Health Sciences Center, El Paso, Texas 79905, USA \\ ${ }^{3}$ Professor and Clinical Professor of Obstetrics and Gynecology and Radiology, Department of Medical Education, Paul L \\ Foster School of Medicine, Texas Tech University Health Sciences Center, 5001 El Paso Drive, El Paso, Texas 79905, USA
}

Correspondence: Sandesh Subramanya, Instructor, Department of Biomedical Sciences Center of Excellence for Infectious Diseases Paul L Foster School of Medicine, Texas Tech University Health Sciences Center, El Paso, Texas 79905, USA e-mail: sandesh.subramanya@ttuhsc.edu

\begin{abstract}
Maternal infections with TORCH group of organisms during pregnancy pose a threat to the fetus in acquisition of congenital abnormalities. Detailed ultrasonographic screening and serological testing provide vital clues to the early diagnosis of these infections in the fetus. We summarize the clinical features associated with $\mathrm{TORCH}$ with special emphasis on the in utero ultrasound-guided diagnosis.

Keywords: TORCH infection, prenatal ultrasound, pregnancy, syphilis, HIV infection.
\end{abstract}

\section{INTRODUCTION}

Intrauterine infections acquired during pregnancy can have varying effects on fetal growth and development. Even if the fetus survives to term, significant morbidity may be noticed in the neonate. A thorough understanding of all intrauterine infections in general, and TORCH group of infections in particular ${ }^{1}$ should be emphasized to the medical students since they constitute one of the most preventable causes of fetal malformations. TORCH group of infections pertain to the "famous five" culprits of the intrauterine infections. ${ }^{2-4}$ TORCH stands for Toxoplasmosis, Other (e.g., Syphillis), Rubella, Cytomegalovirus, and Herpes simplex virus.

Medical students should be able to recognize these presentations in reproductive age group patients, and if possible, provide adequate counseling prior to conception. In fact, TORCH screening (serology and titers) as a part of pregnancy planning or during the first prenatal visit is highly advocated. ${ }^{5-7}$ However, in many instances, a routine fetal ultrasound during pregnancy provides the first clue into the presence of these infections. ${ }^{8-11,31,32}$ Medical students should be aware of the following components for diagnosis of TORCH: (i) Complete prenatal history and thorough physical examination (for diagnosis of previous or current maternal infections), (ii) TORCH agents' titers during first prenatal visit (for diagnosis of previous or current maternal infections) and (iii) fetal ultrasound (for diagnosis of fetal consequences of maternal infection). ${ }^{12}$ In cases of fetal infections, as evidenced by ultrasound as well as serological reports, proactive management can limit further damage to the fetus. Adequate counseling may prevent similar episodes in future pregnancies. Following is the presentation of the integrated approach we have adopted for TORCH group of infections in our curriculum.

\section{TORCH INFECTIONS}

\section{Fetal Toxoplasmosis Syndrome}

The causative agent is Toxoplasma gondii, a ubiquitous parasite found in mammals. Only an active infection acquired between 7-14 weeks results in fetal toxoplasmosis syndrome. Even if the parasite is acquired in the window of 7-14 weeks, the chances of severe fetal infection is $1: 10 .^{13}$ Rest of the fetuses have either subclinical infections, or may escape infection completely.

History and physical examination: A history of contact with feline feces (cat litter boxes or soil) and history of consuming 


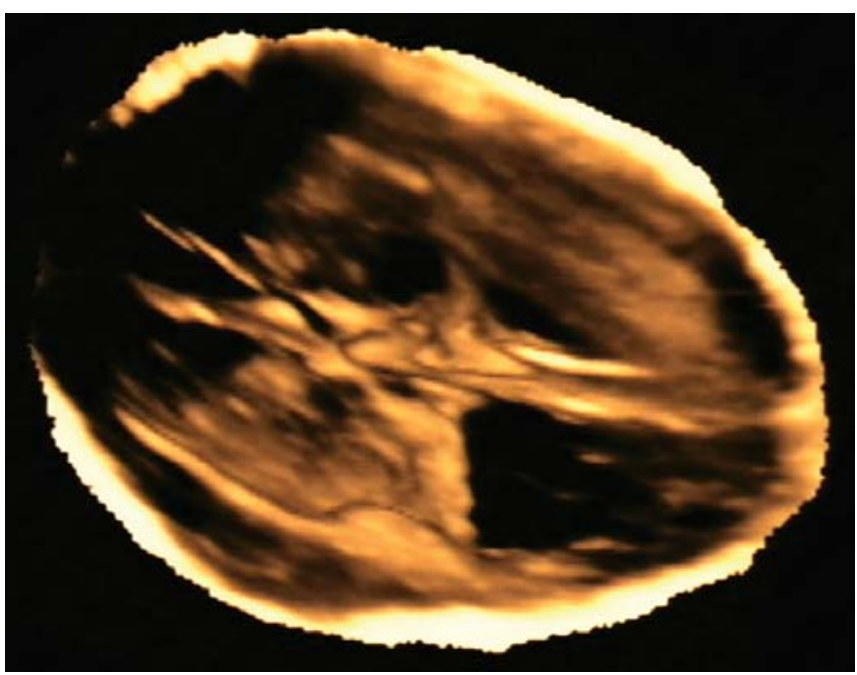

Fig. 1: Three-dimensional ultrasound image of hydrocephalus at 32 weeks' gestation. Serology and titers indicated TORCH infection

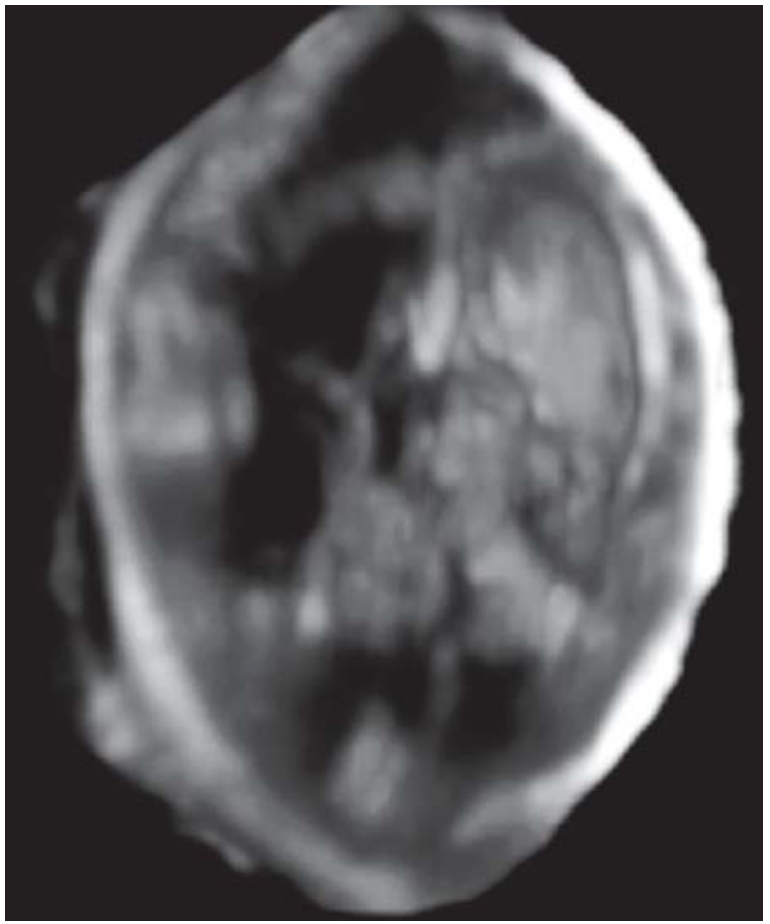

Fig. 2: Hydrocephalus and intracranial calcifications visualized in a fetus with $\mathrm{TORCH}$ infection

undercooked meat should always be enquired. In most adults, Toxoplasmosis remains relatively asymptomatic, but can present as fever, malaise, and lympadenopathy.

Diagnosis: T gondii antibody titers during prenatal visit may be done as a part of TORCH panel. Fetal ultrasound may reveal microcephaly, hydrocephalus, intracranial calcifications (Figs 1 and 2). ${ }^{10,14-16}$ Postpartum complications may include chorioretinitis, seizures secondary to calcifications and mild to severe mental retardation.

\section{Fetal Herpes Simplex Infection}

The causative agents are Herpes Simplex virus type 1 or type 2. While HSV type 1 is still the most common causative agent, it has been closely followed by HSV-type 2 in incidence rates.

History and physical examination: Patient may reveal the history of or present with herpetic rashes in the orogenital area during the pregnancy. Newly acquired primary genital herpes during pregnancy poses the most serious threat for intrauterine and neonatal infection. While the most common mode of transmission is during passage of the baby through the birth canal during labor, instances of intrauterinetransplacental transmission have been noted as well.

Diagnosis: Serology can reveal past or current HSV infections. Fetal ultrasound may reveal intrauterine growth retardation, microophthalmia, microcephaly, hydrancephaly and hepatosplenomagely. ${ }^{12,17}$ Postpartum complications may include cataract and mental retardation.

\section{Congenital Rubella Syndrome}

The causative agent is rubella virus (togavirus family). Primary maternal infection during pregnancy results in fetal rubella syndrome. The incidence rates have dramatically reduced after the introduction of routine rubella vaccination in reproductive age group women.

History and physical examination: Most cases in adults are asymptomatic, but may present with flu-like illness. History of rash that begins in trunk and spreads caudally can be usually elicited. Examination reveals maculopapular rash and cervical lympadenopathy.

Diagnosis: Maternal rubella titers are diagnostic. Fetal ultrasound may reveal cardiac malformations (e.g., patent ductus arteriosus, septal defects), microphthalmia, hepatosplenomegaly and intrauterine growth retardation. ${ }^{10,18}$ Postpartum complications include sensorineural deafness, cataract, meningoencephalitis and mental retardation.

\section{Intrauterine Cytomegalovirus Infection}

The causative agent is cytomegalovirus (Herpes virus family). Primary infection or reactivation during pregnancy is believed to cause fetal CMV infection. Prior exposure to CMV in mothers is known to have protective effect on subsequent infections. 
History and physical examination: Most cases in adults are asymptomatic, but may present with flu-like illness.

Diagnosis: Maternal CMV titers and viral cultures are diagnostic. ${ }^{19}$ Fetal ultrasound may reveal nonimmune intrauterine growth retardation, cerebral ventriculomegaly, microcephaly, fetal hydrops, chorioretinitis, and intracranial calcifications. ${ }^{20-22}$ Although ultrasound abnormalities can predict fetal CMV infection, ${ }^{20}$ fetal brain MRI can highlight additional cerebral features including abnormal gyration, cerebellar hypoplasia, or abnormal signal in white matter. ${ }^{23}$

\section{Intrauterine Syphilitic Infections}

Syphilis is a sexually transmitted disease caused by Trepanoma pallidum (spirochete).

History and physical examination: Primary syphilis presents as painless chancre at the site of inoculation. Secondary syphilis presents as generalized maculopapular rash, generalized lympadenopathy, and condylomata lata (white lesions).

Diagnosis: Antepartum screening and appropriate management can effectively eliminate fetal consequences of maternal syphilis. Direct florescent antibody staining can be used to visualize T. pallidum. VDRL tests can be run to screen for syphilis, if positive, a more specific Treponemal antibody (FTA-ABS) can be performed. Perinatal transmissions can occur in any stage of syphilis, although highest incidences are noted in primary and secondary syphilis. Congenital syphilis is diagnosed by ultrasound which may reveal hydrops placentalis and acute chorioamnionitis. ${ }^{24}$ The fetus may have intrauterine growth retardation and hydrops fetalis. ${ }^{25}$

\section{OTHER INFECTIONS}

\section{Human Immunodeficiency Virus (HIV-1)}

Approximately $7000 \mathrm{HIV}-1$ infected women are in the reproducible age range in United States alone and perinatal transmission of HIV-1 to newborn is as high as $50 \%$.

Diagnosis: Antepartum screening for HIV is routine in most settings. It is performed by ELISA, and if positive, followed up with a highly specific western blot followed by determination of viral loads and CD4 T cell counts. The diagnosis is critical, since treatment with antiretrovirals (e.g., zidovudine) during pregnancy and labor, and to the newborn reduces the risk of transmission by $70 \% .{ }^{26,27}$ Multimodal studies may provide primary clues to the diagnosis of AIDS including placentitis (increase in placental thickness), ${ }^{12}$ brain atrophy ${ }^{28}$ and related complications. ${ }^{29,30}$ Coinfection of HIV with Hepatitis B virus, Hepatitis C virus, Herpes Simplex virus and Human Papilloma virus can additionally lead to fetal infections and anomalies that may be detected during ultrasound. ${ }^{12}$

\section{CONCLUDING REMARKS}

In order to effectively diagnose the fetal consequences of TORCH group of infections, the most reliable and least invasive method is fetal ultrasound. It is then of essence to stress that a good command of what constitutes "normal ultrasound" in all trimesters of pregnancy is absolutely essential for medical students. In our curriculum, we are offering a parallel study of microbiological causes, as well as pathological and radiological findings of intrauterine infections. This offers a unique, complete and integrated perspective to the medical students. A quintessential knowledge of reading and interpreting fetal ultrasound is not only valuable but absolutely necessary for better understanding of TORCH group of infections.

\section{REFERENCES}

1. Epps RE, Pittelkow MR, Su and WP. TORCH syndrome. Semin Dermatol 1995;14:179-86.

2. Fine JD, Arndt KA. The TORCH syndrome: A clinical review. J Am Acad Dermatol 1985;12:697-706.

3. Franca CM, Mugayar LR. Intrauterine infections: A literature review. Spec Care Dentist 2004;24:250-53.

4. Greenough A. The TORCH screen and intrauterine infections. Arch Dis Child Fetal Neonatal Ed 1994;70:F163-65.

5. Isada NB, Paar DP, Grossman JH, 3rd and Straus SE. TORCH infections. Diagnosis in the molecular age. J Reprod Med 1992;37:499-507.

6. Kaur R, Gupta N, Nair D, Kakkar M, Mathur MD. Screening for TORCH infections in pregnant women: A report from Delhi. Southeast Asian J Trop Med Public Health 1999;30:284-86.

7. Newton ER. Diagnosis of perinatal TORCH infections. Clin Obstet Gynecol 1999;42:59-70; quiz 174-75.

8. Crino JP. Ultrasound and fetal diagnosis of perinatal infection. Clin Obstet Gynecol 1999;42:71-80; quiz 174-75.

9. Arda K, Ozdemirel D, Tosun and Olcer T. Postpartum followup of hepatic calcification detected by prenatal ultrasound. JBRBTR 2000;83:231-33.

10. Gerber S, Hohlfeld P. Screening for infectious diseases. Childs Nerv Syst 2003;19:429-32.

11. Joo JG, et al. Etiology, prenatal diagnostics and outcome of ventriculomegaly in 230 cases. Fetal Diagn Ther 2008;24: 254-63.

12. Bailao LA, et al. Ultrasound markers of fetal infection part 1: Viral infections. Ultrasound Q 2005;21:295-308. 
13. Pratlong F, et al. Antenatal diagnosis of congenital toxoplasmosis: evaluation of the biological parameters in a cohort of 286 patients. Br J Obstet Gynaecol 1996;103:552-57.

14. Gay-Andrieu F, et al. Fetal toxoplasmosis and negative amniocentesis: Necessity of an ultrasound follow-up. Prenat Diagn 2003;23:558-60.

15. Nowakowska D, et al. Too late prenatal diagnosis of fetal toxoplasmosis: A case report. Fetal Diagn Ther 2005;20:190-93.

16. Kankova S, Flegr J. Longer pregnancy and slower fetal development in women with latent "asymptomatic" toxoplasmosis. BMC Infect Dis 2007;7:114.

17. Duin LK, et al. Major brain lesions by intrauterine herpes simplex virus infection: MRI contribution. Prenat Diagn 2007;27:81-84.

18. Toma $\mathrm{P}$, et al. Cerebral ultrasound images in prenatal cytomegalovirus infection. Neuroradiology 1989;31:278-79.

19. Nigro G. Maternal-fetal cytomegalovirus infection: From diagnosis to therapy. J Matern Fetal Neonatal Med 2009;22: 169-74.

20. Tongsong T, Sukpan K, Wanapirak C, Phadungkiatwattna P. Fetal cytomegalovirus infection associated with cerebral hemorrhage, hydrops fetalis, and echogenic bowel: Case report. Fetal Diagn Ther 2008;23:169-72.

21. Salmaso R, et al. Early detection by magnetic resonance imaging of fetal cerebral damage in a fetus with hydrops and cytomegalovirus infection. J Matern Fetal Neonatal Med 2007;20:559-61.

22. Abdel-Fattah SA, Bhat A, Illanes S, Bartha JL, Carrington D. TORCH test for fetal medicine indications: Only CMV is necessary in the United Kingdom. Prenat Diagn 2005;25:1028-31.

23. Picone O, Simon I, Benachi A, Brunelle F, Sonigo P. Comparison between ultrasound and magnetic resonance imaging in assessment of fetal cytomegalovirus infection. Prenat Diagn 2008;28:753-58.

24. Raafat NA, et al. Sonographic osseous manifestations of fetal syphilis: A case report. J Ultrasound Med 1993;12:783-85.

25. Hollier LM, Harstad TW, Sanchez PJ, Twickler DM, Wendel GD, Jr. Fetal syphilis: Clinical and laboratory characteristics. Obstet Gynecol 2001;97:947-53.

26. Connor EM, et al. Reduction of maternal-infant transmission of human immunodeficiency virus type 1 with zidovudine treatment. Pediatric AIDS Clinical Trials Group Protocol 076 Study Group. N Engl J Med 1994;331:1173-80.

27. Cooper ER, et al. After AIDS clinical trial 076: The changing pattern of zidovudine use during pregnancy, and the subsequent reduction in the vertical transmission of human immunodeficiency virus in a cohort of infected women and their infants. Women and Infants Transmission Study Group. J Infect Dis 1996;174:1207-11.

28. Marquis JR, Bardeguez AD. Imaging of HIV infection in the prenatal and postnatal period. Clin Perinatol 1994;21:125-47.

29. Henrich W, et al. Antenatal diagnosis of placenta percreta with planned in situ retention and methotrexate therapy in a woman infected with HIV. Ultrasound Obstet Gynecol 2002;20:90-93.

30. Hornberger LK, et al. Cardiac structure and function in fetuses of mothers infected with HIV: The prospective PCHIV multicenter study. Am Heart J 2000;140:575-84.

31. Kurjak A, Chervenak FA. Donald School Textbook of Ultrasound in Obstetrics and Gynecology. Publisher: Taylor and Francis, Inc. (2004).

32. Kurjak A, Chervenak FA. Ultrasonography in Obstetrics and Gynecology (5th ed) Publisher: W Saunders and Co, Inc.2008. 